\title{
Tumor Results Numeric Result in Standard Unit
}

National Cancer Institute

\section{Source}

National Cancer Institute. Tumor Results Numeric Result in Standard Unit. NCI

Thesaurus. Code C117424.

The numerical identifier of a tumor result in standard units. 